\title{
Méthode de mesure de la surface foliaire lésée par Marssonina brunnea (Ell. et Ev.) Magn.
}

\author{
Patricia MAURER, J. PINON, Isabelle GENETET \\ INRA, Laboratoire de Pathologie forestière \\ Centre de Recherches de Nancy, Champenoux, F 54280 Seichamps
}

\begin{abstract}
Résumé
Une méthode a été mise au point afin de quantifier le degré d'infection de feuilles de peuplier par Marssonina brunnea. En décolorant les feuilles par le DMSO, les parties saines deviennent transparentes alors que les nécroses brunes et le halo de tissu chlorotique qui les entoure demeurent opaques. Cette différence de contraste est mise à profit pour mesurer à l'analyseur d'image la surface des tissus infectés.
\end{abstract}

Mots clés: Décoloration, analyse d'image.

\section{Introduction}

L'infection des feuilles et jeunes pousses de peuplier, due à Marssonina brunnea (Ell. et Ev.) Magn. (téléomorphe : Drepanopeziza punctiformis Gremmen), s'exprime par l'apparition de petites taches brunes sur les deux faces du limbe foliaire. Ces taches mesurent environ un millimètre de diamètre et s'entourent progressivement d'un halo chlorotique. Elles peuvent être très nombreuses.

La quantification de l'infection du feuillage fait généralement appel à des méthodes basées sur la fréquence de ces taches. Castellani \& Cellerino (1969) ont proposé de toutes les dénombrer sur des disques prélevés au hasard sur les feuilles. RIDÉ (communication personnelle) affecte visuellement les feuilles d'une note issue d'un barème en classes. Ces deux méthodes, assez bien corrélées (Joannes \& PinON, 1982) conviennent avant tout pour les notations au champ, qui portent sur de grands effectifs. Elles deviennent imprécises lorsque le nombre de feuilles est limité. Dans ce cas l'hétérogénéité de la répartition des taches sur le limbe (accumulations le long de la nervure principale ou au bord du limbe) impose le recours au dénombrement parfois fastidieux de toutes les taches qui seront rapportées à la surface du limbe. Bien que plus précise, cette méthode ignore la dualité des symptômes : le dénombrement rend compte seulement des parties brunes (nécrosées) et omet la zone chlorotique qui les entoure. Pourtant celle-ci est importante en surface (par compa- 
raison aux taches) et traduit une perturbation physiologique de l'hôte. Enfin, il n'est pas obligatoire qu'il y ait proportionnalité entre la densité de taches et la surface de limbe chlorotique ou lésé.

Pour ces raisons nous avons mis au point une méthode plus précise qui permet d'estimer la surface foliaire infectée en tenant compte à la fois des taches nécrotiques et des plages chlorotiques.

\section{Principe}

La méthode est basée sur l'observation d'une différence de densité optique entre les tissus infectés et le reste du limbe. Cette différence est accentuée par une décoloration de la feuille qui autorise ultérieurement son étude à l'analyseur d'image.

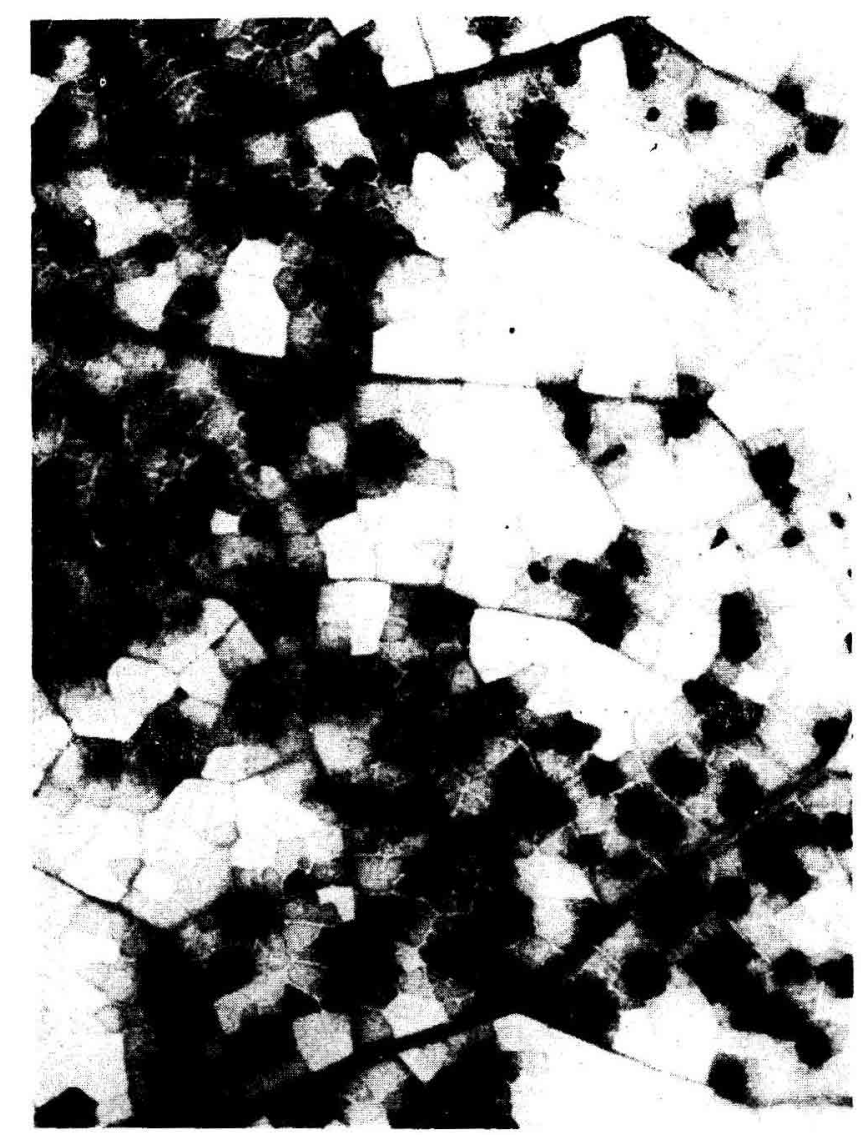

FIG. 1

Portion de limbe décoloré au DMSO : les tissus sains deviennent transparents, les halos chlorotiques gris et les nécroses brunes demeurent sombres.

Part of leaf blade discoloured by DMSO : healthy tissues become transparent, chlorotic halos become gray and brown necrosis remain dark. 
Cette décoloration est acquise en immergeant la feuille dans du diméthylsulfoxyde (DMSO) pur dont Hiscox \& ISRAELSTAM (1979) ont montré l'efficacité pour extraire la chlorophylle. Le DMSO est utilisé à chaud $\left(65\right.$ à $\left.100^{\circ}\right)$ au bain-marie selon des durées variables (20 minutes à une heure) en fonction des caractéristiques de la feuille (dimension, teneur en chlorophylle), ce qui implique un suivi visuel de cette opération. Lorsque la décoloration apparaît totale, la feuille est sortie du DMSO, rincée à l'eau et enfin séchée. Ce procédé respecte les taches brunes attour desquelles apparaissent des zones gris-jaunâtres ayant sensiblement la même taille que les halos chlorotiques. Le tissu vert devient totalement transparent du fait de l'extraction de la chlorophylle (fig. 1).

Les feuilles ainsi préparées sont soumises à l'analyseur d'image (Leitz, Texture Analyse System). Par un réglage de contraste les taches brunes et les halos initialement chlorotiques deviennent opaques (plages blanches sur l'écran) alors que le tissu sain et transparent apparaît sombre sur l'écran (fig. 2). Un programme de calcul introduit dans l'analyseur permet la mesure de l'aire totale des plages opaques (tissus infectés) et celle de l'ensemble du limbe, après un autre réglage de l'appareil.

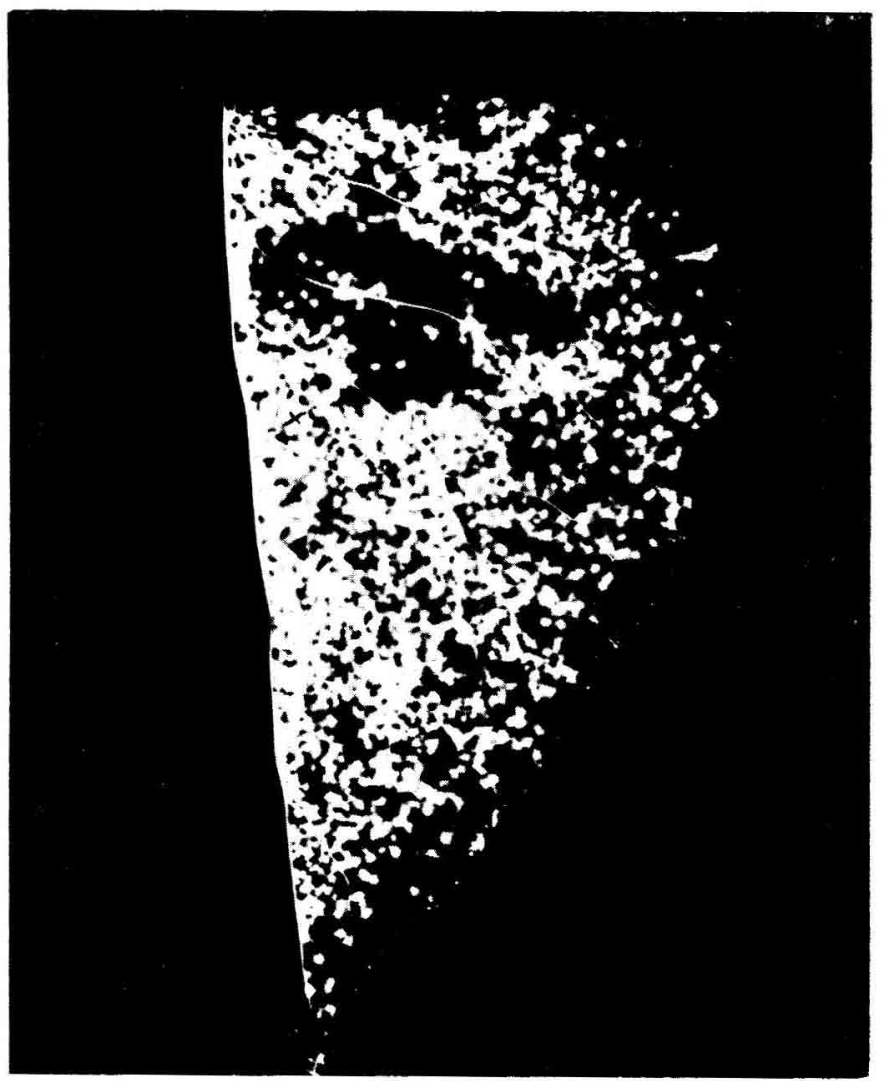

FIG. 2

Aspect d'une demi-feuille décolorée et soumise à l'analyseur d'image, en blanc les tissus infectés.

Aspect of half a leaf discoloured and examined by image analyser, in white infected tissues. 


\section{Conclusion}

En associant la décoloration du feuillage et l'analyse d'image, il devient possible d'estimer le degré d'infection en intégrant l'ensemble de la surface foliaire lésée, notamment lorsque le parasite induit des lésions très petites et donc non mesurables directement. D'autres applications de cette méthode sont envisageables tel que le dénombrement de taches ou la visualisation des zones infectées sur plan film négatif permettant la réalisation de clichés photographiques. Toutefois ceux-ci ne peuvent être analysés par l'appareil déjà cité.

Reçu le 7 février 1986. Accepté le 24 février 1986.

\section{Remerciements}

Les auteurs tiennent à remercier $\mathbf{M}^{\text {mu}}$ Françoise HubER de ses précieux conseils pour l'utilisation de l'analyseur d'image et la préparation du programme de calcul.

\section{Summary}

A method for measuring foliar area injured by Marssonina brunnea

A method has bcen developed in order to quantify the infection of poplar leaves by Marssonina brunnea. When discolouring leaves by DMSO, healthy portions become transparent whilc brown necrosis and chlorotic halos surrounding them remain opaque. This difference in contrast is utilized by an image analyser to measure the area of infected tissues.

\section{Références bibliographiques}

Castellani E., Cellerino G.P., 1969. Cinque anni di osservazioni sul comportamento di vari cloni di pioppo verso la Marssonina brumnea. Cellulosa e Carta, 20, 1-14.

Hiscox J.D., IsRaelstam G.F., 1979. A method for the extraction of chlorophyll from leaf tissue without maceration. Can. J. Bot., 57, 1332-1334.

JoAnnes H., Pinon J., 1982. Comparaison de deux méthodes d'estimation de l'infection de jeunes peupliers par le Marssonina brunnea (Ell. et Ev.) Magn. Eur. J. For. Path., 12, $87-96$. 